\title{
LA RELEVANCIA DE LAS ACTIVIDADES ECOTURÍSTICAS EN COLOMBIA: UNA APROXIMACIÓN ECONÓMICA Y SOCIAL PARA SU DESARROLLO EN EL PARQUE NACIONAL NATURAL LOS NEVADOS. COLOMBIA.
}

\section{The relevance of ecotourism activities in Colombia: an economic and social approach for its development.}

\author{
Lic. Elvia Andrea Lozano Cruz \\ Docente e investigadora. \\ Maestrante en Gestión Socioambiental \\ Universidad de Ciencias Aplicadas y Ambientales (U.D.C.A) Colombia \\ http://dx.doi.org/10.30972/geo.14282745
}

\section{RESUMEN}

El ecoturismo es una práctica y tendencia a nivel mundial, que se desarrolla en zonas alejadas de las urbes, procurando compatibilizar la industria turística con la ecología. EI presente artículo toma en cuenta la relevancia económica del ecoturismo en Colombia en el transcurso del siglo XXI, con el objetivo de analizar su magnitud dentro de las actividades turísticas. Para ello se presentan y analizan datos económicos, a fin de corroborar estas prácticas en la sociedad colombiana de manera implícita o explícita.

Metodológicamente, se realiza una comparación del crecimiento del ecoturismo en lugares como los Parques Nacionales Naturales, mediante datos suministrados por el Ministerio de Comercio y Desarrollo de Colombia, procurando establecer hasta qué punto puede ser considerado como un servicio ecosistémico enmarcado en la conciencia y responsabilidad ambiental.

PALABRAS CLAVES: Ecoturismo; Desarrollo económico; Parques Naturales; Servicios ecosistémicos

\section{ABSTRACT}

Ecotourism is a practice and trend worldwide, which is developed in areas far from the cities, trying to make the tourism industry compatible with ecology. This article takes into account the economic relevance of ecotourism in Colombia during the XXI century, in order to analyze its magnitude within tourism activities. For this purpose, economic data are presented and analyzed in order to corroborate these practices in Colombian society implicitly or explicitly. Methodologically, a comparison of the growth of ecotourism in places like the National Natural Parks, through data provided by the Ministry of Commerce and Development of Colombia, is attempted to establish to what extent it can be considered as an ecosystem service framed in environmental awareness and responsibility.

\section{KEY WORDS}

Ecotourism; Economic development; Natural parks; Ecosystem Services

Publicado en formato digital: Lic. Elvia Andrea Lozano Cruz. LA RELEVANCIA DE LAS ACTIVIDADES ECOTURÍSTICAS EN COLOMBIA: UNA APROXIMACIÓN ECONÓMICA Y SOCIAL PARA SU DESARROLLO EN EL PARQUE NACIONAL NATURAL LOS NEVADOS. COLOMBIA Revista Geográfica Digital. IGUNNE. Facultad de Humanidades. UNNE. Año 14. № 28. Julio - Diciembre 2017. ISSN 1668-5180 Resistencia, Chaco. 


\section{INTRODUCCIÓN}

En Colombia, las prácticas ecoturísticas han ido en auge en las últimas dos décadas, capitalizando la condición de país mega diverso que permite a los turistas tener experiencias distintas y agradables. Por ello, desde el año 2003, el ecoturismo en Colombia ha sido regulado en cuanto al acceso a los sitios que comúnmente se visitan, con el fin de contribuir a un equilibrio entre la preservación de lo natural y la accesibilidad a distintos lugares.

La economía generada por el ecoturismo se puede denominar como un bien, y a su vez se clasifica como un servicio ecosistémico teniendo en cuenta que es un servicio de carácter cultural y se puede enmarcar en lo que hoy se denomina "economía verde", que según EI Programa de las Naciones Unidas para el Medio Ambiente (PNUMA), se define como aquella que da lugar al mejoramiento del bienestar humano e igualdad social, mientras que se reducen significativamente los riesgos medioambientales y la escases ecológica. (2011)

En consecuencia, la Asamblea General de las Naciones Unidas en la resolución (67/223), titulada «Promoción del ecoturismo para la erradicación de la pobreza y la protección del medio ambiente», insta a los Estados Miembros de las Naciones Unidas a adoptar políticas que promuevan el ecoturismo, subrayando sus «efectos positivos en la generación de ingresos, la creación de empleos y la educación y, en consecuencia, en la lucha contra la pobreza y el hambre». Reconoce además que «el ecoturismo crea importantes oportunidades de conservación, protección y uso sostenible de la diversidad biológica y de las zonas naturales, al alentar a las comunidades locales e indígenas de los países en que se encuentran y a los turistas a preservar y respetar el patrimonio natural y cultural». (2013)

Es así que es importante comprender la economía del ecoturismo y discutir sobre los actores sociales beneficiados con los bienes y servicios que provee. GÓMEZ BAGGETHUN (2007) señala que el concepto de los servicios ambientales ha sido objeto de fuertes controversias, al señalarse que favorece una concepción utilitaria y mercantilista de la naturaleza que amenaza con erosionar las motivaciones intrínsecas, simbólicas y culturales que tradicionalmente venían justificando la conservación de los ecosistemas y la biodiversidad.

El sostenido crecimiento del ecoturismo en Colombia como industria ha derivado en la política para el desarrollo de ecoturismo, cuyo objetivo principal es fortalecer y diversificar la actividades ecoturísticas, teniendo como referente esencial su desarrollo sostenible, en razón del cual, ésta debe propender por el uso racional de los recursos, el mejoramiento de la calidad de vida de los pobladores residentes en las regiones y el permanente esfuerzo para proporcionar una oferta competitiva de servicios, en armonía con la diversidad ecológica y cultural. La política propuesta tiene en cuenta que la actividad del turismo involucra varios sectores económicos como servicios ambientales, producción agrícola e

Publicado en formato digital: Lic. Elvia Andrea Lozano Cruz. LA RELEVANCIA DE LAS ACTIVIDADES ECOTURÍSTICAS EN COLOMBIA: UNA APROXIMACIÓN ECONÓMICA Y SOCIAL PARA SU DESARROLLO EN EL PARQUE NACIONAL NATURAL LOS NEVADOS. COLOMBIA Revista Geográfica Digital. IGUNNE. Facultad de Humanidades. UNNE. Año 14. № 28. Julio - Diciembre 2017. ISSN 1668-5180 Resistencia, Chaco. 
industrial, y contempla que, parte de los recursos sean distribuidos de manera equitativa en la población local.

Parques Nacionales de Colombia ha tenido la gran responsabilidad de definir las zonas que pueden ser parte de las prácticas ecoturísticas y las que no, pues su responsabilidad esta en proteger zonas en riesgo y la preservación de la diversidad. Con los lineamientos políticos propuestos, es que surge la guía para la planificación del ecoturismo en Parques Nacionales Naturales de Colombia, la cual, justifica al ecoturismo como una estrategia que mejora o mantiene los valores naturales y culturales, y, además, comparta esta responsabilidad con quienes participen en su desarrollo, propicien al visitante una experiencia particular y estimule alternativas que beneficien económica y ambientalmente a las comunidades locales

\section{MARCO TEÓRICO}

El ecoturismo como actividad económica tiene que ver con el desarrollo de un país, en el caso de Colombia tal actividad se ve enmarcada desde la protección de sus recursos naturales con leyes y normas, en donde la industria turística debe trabajar en equipo con las entidades que protegen las áreas naturales. A continuación se muestra en enfoque que tiene el ecoturismo en Colombia, la economía del turismo en cuanto a su desarrollo en los últimos años, economía del ecoturismo en Parques Nacionales e incremento de visitantes en los Parques Nacionales Naturales.

\section{Ecoturismo}

El ecoturismo tiene como función proteger y cuidar el ambiente, con la intención de no manipular a la naturaleza sino contemplar y admirar su belleza. Conocer a quienes han habitado por siempre en estos ecosistemas, desde las culturas ancestrales, hasta su flora las cualidades curativas y su fauna para respetar el lugar; por lo regular los centros eco turísticos se encuentran en áreas ecológicamente protegidas. Cuando se establecen contactos entre autoridades gubernamentales, habitantes de la comunidad local y profesionistas en turismo se puede lograr sustentabilidad económica y ecológica. Se preserva la naturaleza y se obtiene una derrama económica directa para los habitantes de la localidad rural. (Rebollo, 2012)

Conforme el desarrollo del turismo, deberá hacer de la preservación del ambiente una prioridad. La industria del turismo tendrá que proteger y preservar las atracciones turísticas naturales, el medio ambiente, los ecosistemas frágiles y las culturas vivas de regiones alejadas. De esta manera los turistas continuarán viajando. Cuando el visitante encuentra lugares atractivos y especiales, existe una gran posibilidad que quiera regresar en su próximo asueto. Además, haber tenido una experiencia de viaje satisfactoria, motiva a amigos y parientes para que visiten el mismo lugar.

Publicado en formato digital: Lic. Elvia Andrea Lozano Cruz. LA RELEVANCIA DE LAS ACTIVIDADES ECOTURÍSTICAS EN COLOMBIA: UNA APROXIMACIÓN ECONÓMICA Y SOCIAL PARA SU DESARROLLO EN EL PARQUE NACIONAL NATURAL LOS NEVADOS. COLOMBIA Revista Geográfica Digital. IGUNNE. Facultad de Humanidades. UNNE. Año 14. № 28. Julio - Diciembre 2017. ISSN 1668-5180 Resistencia, Chaco. 
Revista Geográfica Digital. IGUNNE. Facultad de Humanidades. UNNE. . Año 14. № 28.

Julio - Diciembre 2017. Resistencia, Chaco

Es de vital importancia implementar límites de uso para lograr un desarrollo verdaderamente sustentable; por ejemplo, los recorridos en senderos de selva deberán ser limitados al día. Si se recorre el sendero constantemente, no solamente se daña el terreno, también se vuelve ruidoso que aleja a la fauna. La capacidad de carga es un término de turismo que mide cuántos turistas pueden visitar un lugar con el mínimo efecto de contaminar. (Rebollo.2012.p. 8-12). Comprendiendo que la capacidad de carga según la OMT es el nivel de explotación turística que una zona puede soportar asegurando una máxima satisfacción a los visitantes y una mínima repercusión sobre los recursos. Esta noción supone la existencia de limites a los visitantes. OMT (1992). En Parques Nacionales Naturales la Capacidad de carga es de 799 personas por día.

En consecuencia se debe tener en cuenta que El ecoturismo es aquella modalidad turística ambientalmente responsable consistente en viajar o visitar áreas naturales relativamente sin disturbar con el fin de disfrutar, apreciar y estudiar los atractivos naturales (paisaje, flora y fauna silvestres) de dichas áreas, así como cualquier manifestación cultural (del presente y del pasado) que puedan encontrarse ahí, a través de un proceso que promueve la conservación, tiene bajo impacto ambiental y cultural, y propicia un involucramiento activo y socioeconómicamente benéfico de las poblaciones locales. ( citado en Orgaz, 2013)

Para la entidad Parques Nacionales Naturales el ecoturismo debe ser una estrategia que mejore o mantenga los valores naturales y culturales, comparta esta responsabilidad con quienes participen en su desarrollo, propicien al visitante una experiencia particular y estimule alternativas que beneficien económica y ambientalmente a las comunidades locales y a las regiones, a partir de las oportunidades que representan estas áreas protegidas en el país. ( Guia para la planificacion del ecotururismo 2013)

CONSIDERACIONES EN TORNO AL ECOTURISMO: UN ANALISIS DAFO.

\begin{tabular}{|c|c|}
\hline $\begin{array}{l}\text { DEBILIDADES } \\
\text { (ecoturista) }\end{array}$ & $\begin{array}{l}\text { AMENAZAS } \\
\text { (destinos) }\end{array}$ \\
\hline $\begin{array}{l}\text { - Bajo flujo de turistas frente a otras tipologías } \\
\text { turísticas. } \\
\text { - El nivel de pernoctaciones por parte de los } \\
\text { turistas es bajo. }\end{array}$ & $\begin{array}{l}\text { - Inadecuados modelos de marketing. } \\
\text { - Alteración de flora y fauna. } \\
\text { - Lugares alejados de los principales polos } \\
\text { turísticos. } \\
\text { - Elevación de la contaminación. } \\
\text { - Deterioro de la cultura local. } \\
\text { - Baja calidad de la infraestructura. }\end{array}$ \\
\hline $\begin{array}{l}\text { FORTALEZAS } \\
\text { (ecoturista) }\end{array}$ & $\begin{array}{l}\text { OPORTUNIDADES } \\
\text { (destinos) }\end{array}$ \\
\hline $\begin{array}{l}\text { - Elevado nivel de educación. } \\
\text { - Respeto hacia el medio ambiente. } \\
\text { - Grantactorés en actividades turísticas en } \\
\text { - Búsqueda de la auturaleza. } \\
\text { - Mayor disponibilidad de lo locara viajar. }\end{array}$ & $\begin{array}{l}\text { - Desarrollo socioeconómico de las } \\
\text { comunidades locales. } \\
\text { - Creación de empleo y empresas. } \\
\text { - Aprovechamiento sostenible de los recursos } \\
\text { naturales. } \\
\text { - Aumento de los viajes hacia destinos } \\
\text { sostenibles. } \\
\text { - Mejoras de la gestión de las áreas } \\
\text { protegidas. }\end{array}$ \\
\hline
\end{tabular}

Tomado de: Revista en investigación y desarrollo local.

Publicado en formato digital: Lic. Elvia Andrea Lozano Cruz. LA RELEVANCIA DE LAS ACTIVIDADES ECOTURÍSTICAS EN COLOMBIA: UNA APROXIMACIÓN ECONÓMICA Y SOCIAL PARA SU DESARROLLO EN EL PARQUE NACIONAL NATURAL LOS NEVADOS. COLOMBIA Revista Geográfica Digital. IGUNNE. Facultad de Humanidades. UNNE. Año 14. № 28. Julio - Diciembre 2017. ISSN 1668-5180 Resistencia, Chaco. En: http://hum.unne.edu.ar/revistas/geoweb/default.htm 
Revista Geográfica Digital. IGUNNE. Facultad de Humanidades. UNNE. . Año 14. № 28.

Julio - Diciembre 2017. Resistencia, Chaco

\section{Economía del Turismo en Colombia}

El desarrollo de un país puede suponer múltiples caminos u opciones, una de ellos es el turismo en general que ha experimentado un crecimiento exponencial, pues tiene con que competir por su riqueza en aspectos culturales, gastronómicos y naturales.

En Colombia se propuso la actividad turística desde 1931 ya que se vio como ayuda al crecimiento económico para el país y sabía lo que tenía para ofrecer desde ese momento, aun así, no era de gran avance este proyecto que fue progresando con las ideas de tener una conciencia ambiental a partir de realizar recorridos por lugares naturales. En 2002 con las políticas propuestas en ese entonces es que en auge del turismo empieza a crecer de manera más rápida.

Según el Ministerio de Comercio, Industria y Turismo (MCIT) de Colombia, el sector turístico generó durante la década de 1990 un ingreso de divisas al país similar al producido por las exportaciones tradicionales. Alcanzó entre el $20 \%$ y $30 \%$ del total de las divisas aportadas por las exportaciones y el equivalente al $3 \%$ del Producto Interno Bruto; la actividad turística contribuyó entre 1999-2004 en promedio con 2,3\% del PIB. En el año 2006 llegaron al país 1.978 .593 viajeros extranjeros lo que representó un crecimiento del $48.10 \%$ frente al 2005 cuando llegaron 1.335.946 turistas, generando así un ingreso al país de US\$1.455 millones por concepto de turismo, US\$ 337 más que en 2005 cuando ingresaron en este mismo período US\$ 1.118 millones, presentándose un crecimiento del $30.13 \%$ con respecto al mismo período de 2005 (Cifras al tercer trimestre de 2006 de la Balanza de Pagos, Banco de la República). (Such et.al. 2009)

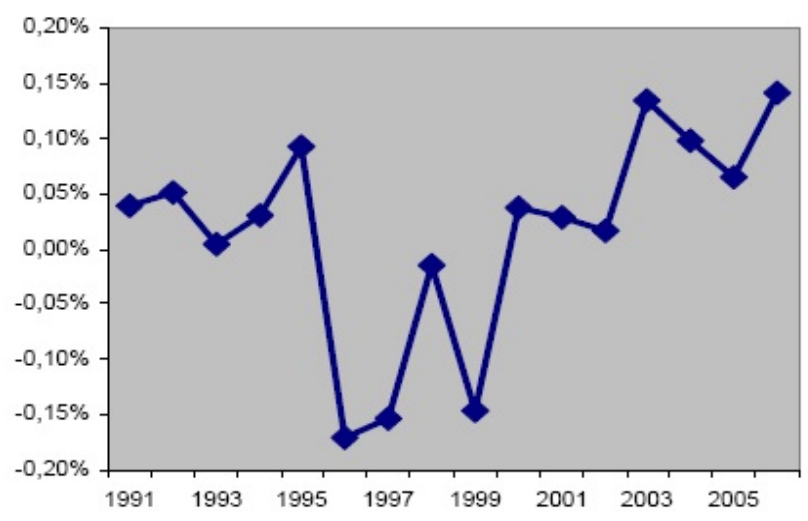

Figura 1 Contribución turismo al crecimiento económico

Fuente: Elaboración propia con base en datos del Banco Central de Colombia y del Departamento Administrativo Nacional de Estadística.

Tomado de: Turismo y crecimiento económico: Un análisis empírico de Colombia

Se puede apreciar en la figura que en Colombia el turismo presenta una caída del turismo en los años 1996- 1999 debido al valor del producto interno bruto PIB en tales años, en relación a la industria asociada en hoteles y restaurantes.

Publicado en formato digital: Lic. Elvia Andrea Lozano Cruz. LA RELEVANCIA DE LAS ACTIVIDADES ECOTURÍSTICAS EN COLOMBIA: UNA APROXIMACIÓN ECONÓMICA Y SOCIAL PARA SU DESARROLLO EN EL PARQUE NACIONAL NATURAL LOS NEVADOS. COLOMBIA Revista Geográfica Digital. IGUNNE. Facultad de Humanidades. UNNE. Año 14. № 28. Julio - Diciembre 2017. ISSN 1668-5180 Resistencia, Chaco.

En: http://hum.unne.edu.ar/revistas/geoweb/default.htm 
Revista Geográfica Digital. IGUNNE. Facultad de Humanidades. UNNE. . Año 14. № 28.

Julio - Diciembre 2017. Resistencia, Chaco

\section{Economía del Ecoturismo en Parques Nacionales Naturales}

Los Parques Nacionales Naturales son un lugar que están siendo visitado más cada año lo cual provee servicios ecosistémicos en el marco de lo cultural, y a su vez se recogen incentivos económicos, pero también se tiene en cuenta que son zonas de protección y conservación natural un recurso de alta protección es el recurso hídrico.

En coordinación con las autoridades correspondientes, Parques Nacionales Naturales de Colombia impulsará el turismo sostenible en las zonas amortiguadoras a las áreas del Sistema, con el fin de dinamizar la economía de estas zonas y de generar beneficios a las comunidades locales asociado al ecoturismo.

El Sistema está compuesto por 49 áreas protegidas, cubre 10 millones de hectáreas y constituye $9 \%$ del territorio nacional. Sus áreas protegidas, 16 de las 49 , son fundamentales para la producción y regulación hídrica del país al contar con cuatro de las seis estrellas hidrográficas más importantes del país. El SPNN contiene, asimismo, dos de las más importantes zonas de alta biodiversidad mundial: el corredor del Chocó Biogeográfico y los Bosques Amazónicos. Por último, el SPNN recibe un promedio de visitantes por año igual a 433.135 y cuenta con una capacidad instalada para alojar 1.774 visitantes. (Carriazo et al. 2003).

Colombia al ser el segundo país en biodiversidad en términos de su extensión, ha venido tomando el ecoturismo como desarrollo económico se toma en cuenta que como se ha tomado por parte de los economistas es una necesidad de valorar los recursos ambientales a desarrollar modelos económicos cuyo objetivo es estimar los beneficios económicos derivados de conservar los recursos naturales y de preservar la calidad ambiental.

La valoración de los aportes del SPNN a la economía colombiana se puede basar en la aplicación de las técnicas de valoración ambiental, pero implicaría estudios de gran envergadura con altos costos económicos y un lapso de tiempo prolongado. Una alternativa a emprender estudios de valoración económica es aprovechar el conjunto de investigaciones existentes para realizar una transferencia de beneficios. La transferencia de beneficios es un instrumento desarrollado por los economistas ambientales con el cual se puede estimar los beneficios provistos por el SPNN a un bajo costo y en un periodo de tiempo razonable. (Carriazo et al. 2003)

\section{Visitas a los Parques Nacionales Naturales.}

A continuación, se presentan las visitas que han tenido los Parque Nacionales Naturales desde el año 2006 a 2017.

Publicado en formato digital: Lic. Elvia Andrea Lozano Cruz. LA RELEVANCIA DE LAS ACTIVIDADES ECOTURÍSTICAS EN COLOMBIA: UNA APROXIMACIÓN ECONÓMICA Y SOCIAL PARA SU DESARROLLO EN EL PARQUE NACIONAL NATURAL LOS NEVADOS. COLOMBIA Revista Geográfica Digital. IGUNNE. Facultad de Humanidades. UNNE. Año 14. N 28. Julio - Diciembre 2017. ISSN 1668-5180 Resistencia, Chaco.

En: http://hum.unne.edu.ar/revistas/geoweb/default.htm 


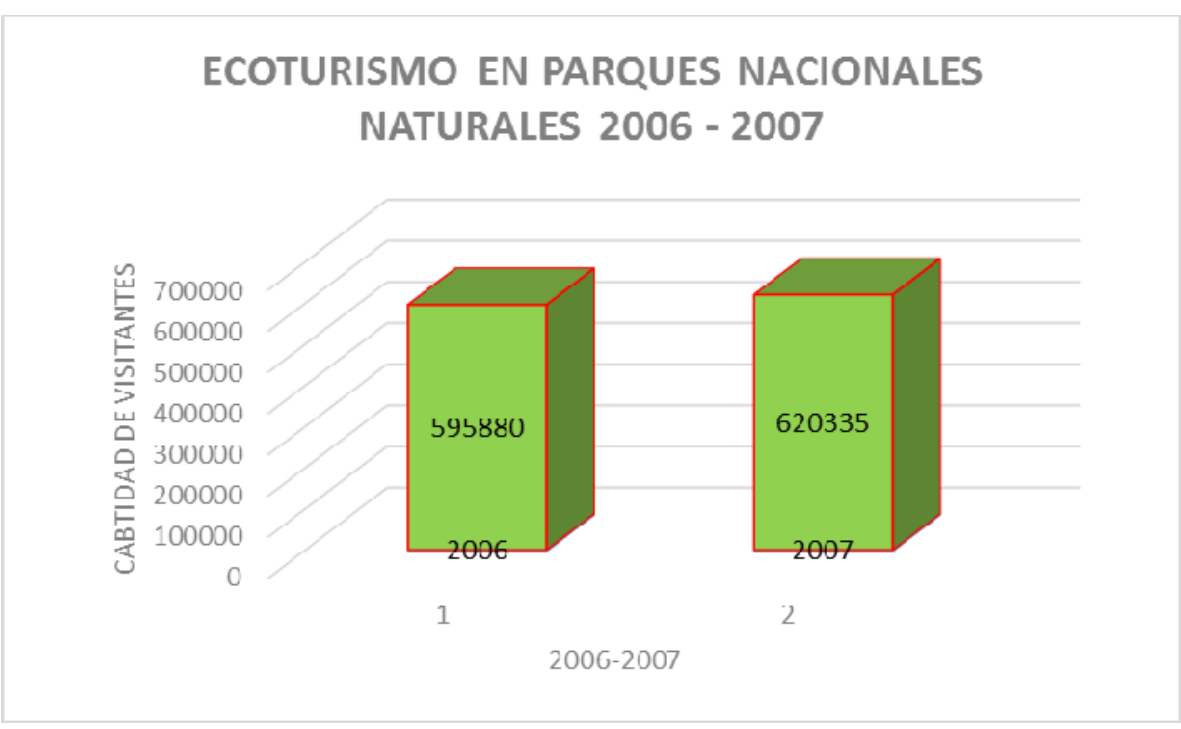

Adaptado de informe de turismo 2006-2007

El el 2007 los parque nacinales naturales fueron vistados por 620.335 personas, con un incremento del $4.1 \%$ frente al año anterior 2006.

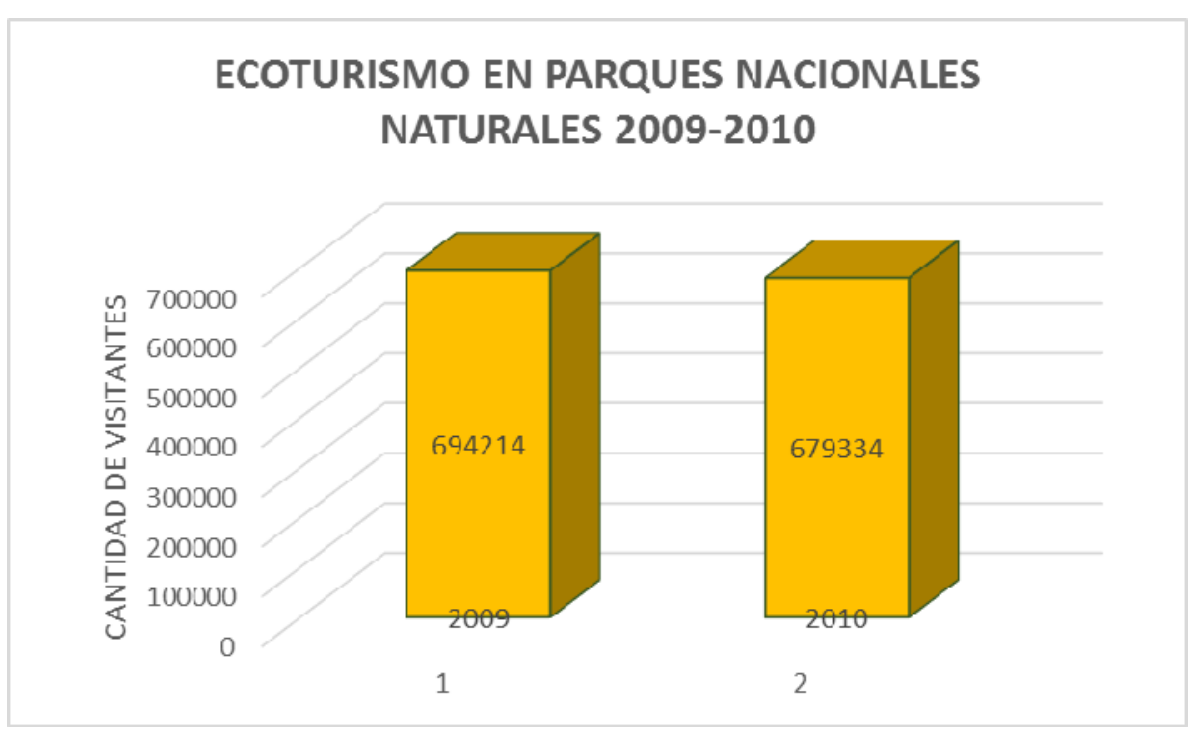

Adaptado de informe de turismo 2009-2010

Para el preriodo de tiempo que se presenta en la grafica enterior es importante tener en cuenta que del año 2009 a 2010 bajaron el numero de visitas en un $2 \%$ en relacion en ese periodo de tiempo debido a que algunos Parques Nacionales han tenido que restringir sus visitas por la regulacion que deben en cuanto a las alertas que se puedan presentar en los lugares naturales. 
Revista Geográfica Digital. IGUNNE. Facultad de Humanidades. UNNE. . Año 14. № 28.

Julio - Diciembre 2017. Resistencia, Chaco

ECOTURISMO EN PARQUES NACIONALES NATURALES

2016-2017

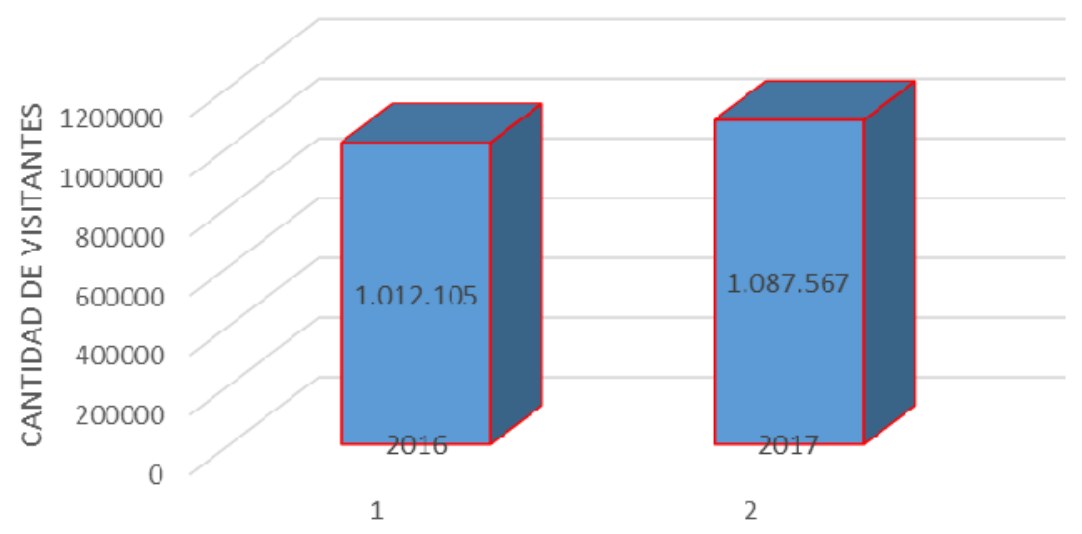

Adaptado de informe de turismo 2016-2017

El crecimiento exponencial del ecoturismo en los Parques Nacionales Naturales para el periodo 2016 - 2017, evidencia que esta práctica va en aumento y que representa para la economía colombiana un índice de crecimiento.

El plan sectorial de turismo 2002-2006 "Turismo para un nuevo país", contempla una visión 2020 para el sector en la cual el ecoturismo, siguiendo las tendencias internacionales, es uno de los siete productos fundamentales en los cuales el país concentrará sus esfuerzos en las próximas décadas"

La "política nacional para el desarrollo del ecoturismo", divulgada en el 2004, contiene los elementos fundamentales para el desarrollo de esta actividad, proponiéndose fortalecer y diversificar la actividad ecoturística teniendo como referente esencial su desarrollo sostenible, y una oferta competitiva de servicios, en armonía con la diversidad ecológica y cultural (citado en Molina 2011)

\section{Empresas de Ecoturismo en Colombia.}

En Colombia el turismo en general se ha tomado como una forma de desarrollo económico, en consecuencia, han nacido empresas o fundaciones que se encargan de incentivar dichas actividades con el fin de generar cultura ambiental.

Aparte de entidades gubernamentales como lo es Parques Nacionales Naturales de Colombia, también existen otro tipo de empresas con las cuales se denominan no gubernamentales como lo es FUNDACION NEVADOS del Municipio Murillo Tolima Colombia en donde su tiene como objetivo es ser la organización no gubernamental más

Publicado en formato digital: Lic. Elvia Andrea Lozano Cruz. LA RELEVANCIA DE LAS ACTIVIDADES ECOTURÍSTICAS EN COLOMBIA: UNA APROXIMACIÓN ECONÓMICA Y SOCIAL PARA SU DESARROLLO EN EL PARQUE NACIONAL NATURAL LOS NEVADOS. COLOMBIA Revista Geográfica Digital. IGUNNE. Facultad de Humanidades. UNNE. Año 14. № 28. Julio - Diciembre 2017. ISSN 1668-5180 Resistencia, Chaco. 
reconocida a nivel nacional e internacional en términos de conservación y protección ambiental y turismo sostenible.

La Fundación Nevados se denomina sin ánimo de lucro, pues los recursos económicos que obtiene por los paquetes turísticos que ofrecen son invertidos en campañas ambientales para el municipio, aun así, con su proyección en cuanto a generar más llegada de turísticos hace que se active la economía del municipio.

\section{Conclusiones}

El ecoturismo debe seguir siendo una práctica de motivación hacia la conservación ambiental como hasta el momento lo ha sido, que permite a las personas tener una realcion mas cercana con a naturaleza y desde allí comprender la importancia de la conservación de nuestros recursos naturales.

Al realizar las practicas de ecoturismo es importante tener en cuenta las capacidades de carga de los lugares naturales, pues si bien es cierto que hace que la economía de un lugar se active por medio del desarrollo social y cultural de los lugares cercanos, también es importante tener en cuenta que el lucro viene de lugares que nos proveer recursos naturales que se deben proteger.

La relevancia de la economía del ecoturismo en Colombia es un tema de gran importancia para poder comprender parte de su desarrollo en temas ambientales y como propone una posible economía sostenible desde practicas ambientales que general algún lucro económico para el país.

Colombia es uno de países con biodiversidad del mundo por ello puede entrar con gran fuerza y estar en la cabeza de países que le apuestan ecoturismo como economía, teniendo en cuenta que en la ultima década se ha venido presentando un crecimiento exponencial del eecoturismo en los Parques Nacionales Naturales.

El trabajo que hace Parques Nacionales Naturales en cuanto al ecoturismo es bien regulado y genera confianza en que la protección y conservación de nustros lugares naturales están siendo cuidados de manera adecuada.

Publicado en formato digital: Lic. Elvia Andrea Lozano Cruz. LA RELEVANCIA DE LAS ACTIVIDADES ECOTURÍSTICAS EN COLOMBIA: UNA APROXIMACIÓN ECONÓMICA Y SOCIAL PARA SU DESARROLLO EN EL PARQUE NACIONAL NATURAL LOS NEVADOS. COLOMBIA Revista Geográfica Digital. IGUNNE. Facultad de Humanidades. UNNE. Año 14. N 28. Julio - Diciembre 2017. ISSN 1668-5180 Resistencia, Chaco. 
Revista Geográfica Digital. IGUNNE. Facultad de Humanidades. UNNE. . Año 14. № 28.

Julio - Diciembre 2017. Resistencia, Chaco

\section{Bibliografía}

- Ambiente, M. d. (2010). Ley de areas protegidas.

- Brida, M. (2011). Impactos del turismo sobre el crecimiento económico y el desarrollo. El caso de los principales destinos de Colombia. Pasos, Revista de Turismo y Patrimonio Cultural, 1-9.

- Carriazo, I. G. (2003). Vloracion de los servicion provistos, por el sistema de Parques Nacionales Naturales.

- Gines de Rus, C. L. (1997). Economia del ecoturismo, un panorama . Revista de economia Aplicada, 93.

- MINCOMERCIO, INDUSTRIA Y TURISMO . (Octubre de 2017). MINCOMERCIO, INDUSTRIA Y TURISMO. Recuperado el 29 de Octubre de 2017, de http://www.mincit.gov.co/publicaciones/16590/informes_de_turismo

- Ministerio de Ambiente. (2017). Aporte de los Parques Nacionales Naturales al desarrollo Socio-economico de Colombia . Colombia.

- Ministerio de Desarrollo Economico . (1996). Ley del Turismo . Bogotá.

- Molina, C. (2011). ECOTURISMO EN COLOMBIA: UNA RESPUESTA A NUESTRA INVALUABLE RIQUEZA NATURAL. Revista en Investigacion y desarrollo local.

- Parques Nacionales Naturales . (2013). Guia para la planificacion del ecotursmo en Parques Nacionales Naturales de Colombia. Colombia .

- Rebollo, s. N. (2012). Ecoturismo. Mexico : Red tercer milenio .

- soyECOlombiano. (2012). Ecoturismo, un buen futuro con turismo sostenible. soyecolombiano , 1-8.

- Such, D. e. (2009). Turismo y Creciemiento en Colombia, un analisis empirico en Colombia. . Estudios y perspectivas en Turismo .

- Vanegas, M. (2006). Ecoturismo Intrumento de Desarrollo Sostenible. Medellin , Colombia . 